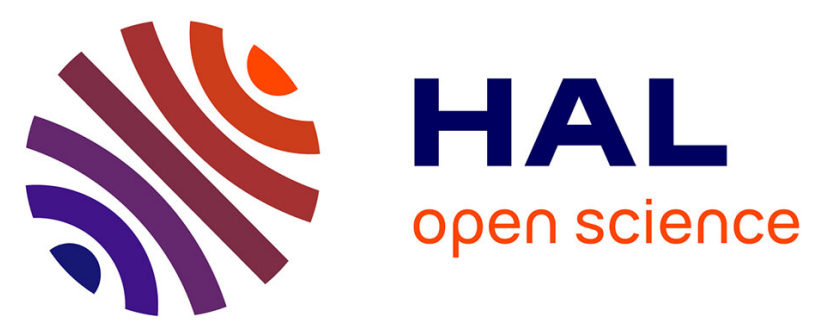

\title{
Transfer matrices-based output-only statistical damage localization and quantification on a frame structure
}

\author{
Md Delwar Hossain Bhuyan, Saeid Allahdadian, Michael Döhler, Yann \\ Lecieux, Laurent Mevel, Franck Schoefs, Carlos Ventura
}

\section{- To cite this version:}

Md Delwar Hossain Bhuyan, Saeid Allahdadian, Michael Döhler, Yann Lecieux, Laurent Mevel, et al.. Transfer matrices-based output-only statistical damage localization and quantification on a frame structure. IWSHM - 11th International Workshop on Structural Health Monitoring, Sep 2017, Stanford, United States. 10.12783/shm2017/13964 . hal-01589536

\section{HAL Id: hal-01589536 \\ https://hal.inria.fr/hal-01589536}

Submitted on 18 Sep 2017

HAL is a multi-disciplinary open access archive for the deposit and dissemination of scientific research documents, whether they are published or not. The documents may come from teaching and research institutions in France or abroad, or from public or private research centers.
L'archive ouverte pluridisciplinaire HAL, est destinée au dépôt et à la diffusion de documents scientifiques de niveau recherche, publiés ou non, émanant des établissements d'enseignement et de recherche français ou étrangers, des laboratoires publics ou privés. 
Title: Transfer matrices-based output-only statistical damage localization and quantification on a frame structure

\author{
Authors : Md Delwar Hossain Bhuyan \\ Saeid Allahdadian \\ Michael Döhler \\ Yann Lecieux \\ Laurent Mevel \\ Franck Schoefs \\ Carlos Ventura
}




\begin{abstract}
Damage localization in civil or mechanical structures is a subject of active development and research. In this paper, vibration measurements and a finite element model are used to locate loss of stiffness in a steel frame structure at the University of British Columbia. Damage localization is not very well developed so far and few methods show promising properties in practice. Among them, the Stochastic Dynamic Damage Locating Vector (SDDLV) approach has interesting capabilities. In this paper, it is compared to a sensitivity based approach developed by the authors. Both approaches have in common to be built on the estimated transfer matrix difference between reference and damaged states. Both methods are tested for localization and quantification on the Yellow Frame structure at the University of British Columbia.
\end{abstract}

\title{
INTRODUCTION
}

The detection, localization and quantification of damages based on measured vibration data are fundamental tasks for structural health monitoring (SHM) to allow an automated damage diagnosis [1]. We consider the case of output-only vibration measurements of a structure subject to ambient excitation. Damages are considered as changes in the structural stiffness.

The Stochastic Dynamic Damage Locating Vector (SDDLV) approach is a vibration based damage localization method using both finite element (FE) information of the structure in the reference state, and modal parameters estimated from measurements in both damage and reference states of the system [2]. It is a theoretically sound method that evaluates the transfer matrix difference between reference and damaged states of a structure, without updating the FE model. In previous works, the deterministic SDDLV approach has been extended with a statistical framework, taking into account the uncer-

Md Delwar Hossain Bhuyan, Michael Döhler, Laurent Mevel - Inria, I4S / IFSTTAR, COSYS, SII, Campus de Beaulieu, 35042 Rennes, France.

Saeid Allahdadian, Carlos Ventura - Department of Civil Engineering, University of British Columbia, 6250 Applied Science Lane, Vancouver, BC V6T 1Z4, Canada.

Yann Lecieux, Franck Schoefs - GeM, Université de Nantes, UMR CNRS 6183, 2 rue de la Houssinière, 44322 Nantes, France. 
tainties in the measurement data [3]. Further robustness has been achieved through a statistical aggregation of results for different parameters of the method, notably lifting a constraint on the necessary number of sensors $[4,5]$. In previous works, the SDDLV as an output-only method was only considered for damage localization, but not for damage quantification that required input information in related works [6]. In this paper, both damage localization and quantification are investigated based on the transfer matrix difference in a sensitivity-based approach [7] using output-only data. A damage-sensitive parameterization of the system is used, referring e.g. to the element stiffnesses in a finite element model. Through sensitivity analysis on the transfer function matrices with respect to this parameterization, damage is first localized by statistical tests, and then damage is quantified only for the damaged element by estimating the parameter change.

A similar sensitivity-based statistical framework has been successful for damage localization using a subspace residual, recently shown in $\mathrm{PhD}$ thesis [8], where it has been applied in a real test case of a four-story steel frame structure, the Yellow Frame at the University of British Columbia [9]. This case study was also subject of previous endeavors involving the deterministic version of the SDLV approach [10]. In the current paper, the proposed statistical approaches based on the SDDLV and on the transfer matrix sensitivities are demonstrated on the same structure, with the overall goal to obtain reliable damage assessment methods for real, complex structures.

\section{MODELS}

The behavior of a mechanical structure is assumed to be described by a linear timeinvariant (LTI) dynamic system

$$
M \ddot{\mathcal{X}}(t)+C \dot{\mathcal{X}}(t)+K \mathcal{X}(t)=f(t)
$$

where $M, C, K \in \mathbb{R}^{d \times d}$ are the mass, damping and stiffness matrices, respectively, $t$ indicates continuous time and $\mathcal{X} \in \mathbb{R}^{d}$ denotes the displacements at the $d$ degrees of freedom (DOF) of the structure. The external force $f(t)$ is not measurable and modeled as white noise. Let the dynamic system (1) be observed at $r$ coordinates. Since $f(t)$ is unmeasured, it can be substituted with a fictive force $e(t) \in \mathbb{R}^{r}$ acting only in the measured coordinates and that regenerates the measured output. Furthermore, defining $x=\left[\begin{array}{ll}\mathcal{X} & \dot{\mathcal{X}}\end{array}\right]^{T}$, this leads to the corresponding continuous-time state-space model

$$
\left\{\begin{array}{l}
\dot{x}(t)=A x(t)+B e(t) \\
y(t)=C x(t)+D e(t)
\end{array}\right.
$$

with state $x \in \mathbb{R}^{n}$, output $y \in \mathbb{R}^{r}$, state transition matrix $A \in \mathbb{R}^{n \times n}$ and output matrix $C \in \mathbb{R}^{r \times n}$, where $n=2 d$ is the system order and $r$ is the number of outputs. The input influence and direct transmission matrices are $B \in \mathbb{R}^{n \times r}$ and $D \in \mathbb{R}^{r \times r}$ respectively.

\section{DAMAGE LOCALIZATION AND QUANTIFICATION}

Starting point of the SDDLV-based damage localization approach is the transfer matrix difference between reference and damaged states. The transfer function $G(s) \in$ 
$\mathbb{C}^{r \times r}$ of the system at the sensor coordinates can be derived as [2]

$$
G(s)=R(s) D \quad \text { where } \quad R(s)=C(s I-A)^{-1}\left[\begin{array}{c}
C A \\
C
\end{array}\right]^{\dagger}\left[\begin{array}{l}
I \\
0
\end{array}\right]
$$

under the condition that the system order satisfies $n \leq 2 r$. Note that $R(s)$ can be computed from output-only system identification, e.g. using subspace methods [4], while matrices $B$ and $D$ cannot be identified for the computation of $G(s)$. The difference between the transfer matrices in both damaged (variables with tilde) and healthy states is $\delta G(s)=\tilde{G}(s)-G(s)$. Based on this matrix, the subsequent damage assessment depends in particular on the sensor locations and positions and the value of $s$. Damage is considered as changes in the stiffness of system (1), while mass remains unchanged.

\section{Localization with SDDLV Approach}

In the SDDLV approach, a vector in the null space of the difference between the transfer matrices of the healthy and damaged systems is obtained. Since the matrices $\delta G(s)$ and $\delta R(s)^{T}=\tilde{R}(s)^{T}-R(s)^{T}$ have the same null space due to $\tilde{D}=D$ [2], the desired load vector $v(s) \in \mathbb{C}^{r}$ can be obtained from the null space of $\delta R(s)^{T}$ using a singular value decomposition (SVD). Thus it can be estimated entirely on measurements in healthy and damaged states. It has been shown that when applying this load vector to the FE model of the healthy structure, then the resulting stress field $S(s)$ is zero at the damaged element. The stress is stacked in a vector and yields a linear relationship

$$
S(s)=\mathcal{L}_{\text {model }}(s) v(s)
$$

defined through the FE model of the healthy state. To decide if the estimated stress components are zero, the covariance of the stress $\Sigma_{S}$ has been estimated in [3-5]. Then, an appropriate hypothesis test is performed on each structural element $t$ by selecting the respective stress components $S_{t}$ in $S(s)$ as well as the respective covariance submatrix $\Sigma_{t}$ of $\Sigma_{S}$, and computing the test statistic

$$
\chi_{t}^{2}=S_{t}^{T} \Sigma_{t}^{-1} S_{t}
$$

for each structural element $t$ tested for damage. Since stress over damaged elements is zero in theory, potential damage is located in elements $t$ corresponding to the lowest values of $\chi_{t}^{2}$ among all elements. Robustness of the localization is achieved by evaluating the stress in (4) for different Laplace variables $s$ and for different mode sets when computing the null space vector $v$, and aggregating the results for each element in $(5)[4,5]$.

\section{Localization and Quantification with Senstivity-Based Approach}

The sensitivity-based approach evaluates a parameterized residual vector $\zeta$ whose mean is zero in the healthy state and that deviates from zero in the damaged state. The parameter vector $\theta \in \mathbb{R}^{l}$ describes the monitored system in the current state, with $\theta_{0}$ in the reference system. It is convenient to choose $\theta$ as the collection of stiffness parameters of the elements of the structure, where $\theta_{0}$ is obtained from a finite element model.

In [7], a statistical framework has been set up for Gaussian residual vectors parametrized by $\theta$ with the purpose to decide which parts of $\theta$ have changed (for damage localization) 
and then to estimate this change (for damage quantification). The Gaussian residual vector $\zeta \in \mathbb{R}^{h}$ is computed from the measurements of the system and needs to satisfy

$$
\zeta \sim \begin{cases}\mathcal{N}(0, \Sigma) & \text { in reference state } \\ \mathcal{N}(\mathcal{J} \delta, \Sigma) & \text { in damaged state }\end{cases}
$$

where $\delta=\sqrt{N}\left(\theta-\theta_{0}\right) \in \mathbb{R}^{l}$ is an unknown change, $N$ is the data length used for the computation of $\zeta$, the sensitivity matrix $\mathcal{J} \in \mathbb{R}^{h \times l}$ of the residual with respect to $\theta$ has full column rank and the residual covariance matrix $\Sigma \in \mathbb{R}^{h \times h}$ is positive definite.

Based on the transfer matrix in the previous section, such a residual is defined as

$$
\zeta \stackrel{\text { def }}{=} \sqrt{N} \operatorname{vec}\left(\tilde{R}(s)^{T}-R(s)^{T}\right)_{\text {re }},
$$

where $(\cdot)_{\text {re }}$ denotes the stacked real-valued vector $q_{\text {re }} \stackrel{\text { def }}{=}\left[\begin{array}{l}\Re(q) \\ \Im(q)\end{array}\right]$ for any vector $q$ with $\Re(\cdot)$ and $\Im(\cdot)$ denoting the real and imaginary parts, respectively, and $\operatorname{vec}(\cdot)$ denotes the column stacking vectorization operator. Note that the mean $\mathcal{J} \delta$ of the residual follows from a first-order Taylor approximation of $\zeta$, based on

$$
\operatorname{vec}\left(\tilde{R}(s)^{T}\right)_{\mathrm{re}} \approx \operatorname{vec}\left(R(s)^{T}\right)_{\mathrm{re}}+\mathcal{J}\left(\theta-\theta_{0}\right) .
$$

The covariance of the quantity $\operatorname{vec}\left(\tilde{R}(s)^{T}-R(s)^{T}\right)_{\text {re }}$ is derived in detail in [4], as well as the sensitivity of $\operatorname{vec}(R(s))$ with respect to the modal parameters. To obtain the required sensitivity matrix $\mathcal{J}$, the derivative of the modal parameters with respect to the structural parameters is needed in addition, which is described in detail in [11].

Note that the transfer matrix can be evaluated for several Laplace variables $s_{i}, i=$ $1, \ldots, n_{s}$. Then, the residual can be defined as

$$
\zeta \stackrel{\text { def }}{=}\left[\begin{array}{c}
\operatorname{vec}\left(\tilde{R}\left(s_{1}\right)^{T}-R\left(s_{1}\right)^{T}\right)_{\mathrm{re}} \\
\vdots \\
\operatorname{vec}\left(\tilde{R}\left(s_{n_{s}}\right)^{T}-R\left(s_{n_{s}}\right)^{T}\right)_{\mathrm{re}}
\end{array}\right] .
$$

The respective sensitivity matrices $\mathcal{J}$ are stacked analogously, and the joint covariance is detailed in $[4,5]$.

\section{DAMAGE LOCALIZATION TESTS}

For damage localization it has to be decided which parts of vector $\delta$ are non-zero, i.e. which parts of the parameter vector are changed. The structural elements corresponding to the changed parameters are thus damaged. To this end, each component of $\delta$ will be tested one after another. Denote the component to be tested by $\delta_{t}$, and the remaining complementary components by $\delta_{C}$, such that

$$
\delta=\left[\begin{array}{l}
\delta_{t} \\
\delta_{C}
\end{array}\right]
$$

Then, $\delta_{t}=0$ is tested against $\delta_{t} \neq 0$. Following (10), the sensitivity matrix $\mathcal{J}$ and the Fisher information matrix $F=\mathcal{J}^{T} \Sigma^{-1} \mathcal{J}$ are analogously arranged as

$$
\mathcal{J}=\left[\begin{array}{ll}
\mathcal{J}_{t} & \mathcal{J}_{C}
\end{array}\right], F=\left[\begin{array}{ll}
F_{t, t} & F_{t, C} \\
F_{C, t} & F_{C, C}
\end{array}\right]=\left[\begin{array}{cc}
\mathcal{J}_{t}^{T} \Sigma^{-1} \mathcal{J}_{t} & \mathcal{J}_{t}^{T} \Sigma^{-1} \mathcal{J}_{C} \\
\mathcal{J}_{C}^{T} \Sigma^{-1} \mathcal{J}_{t} & \mathcal{J}_{C}^{T} \Sigma^{-1} \mathcal{J}_{C}
\end{array}\right]
$$


Sensitivity tests: Assuming that $\delta_{C}=0$ for testing $\delta_{t}=0$ against $\delta_{t} \neq 0$, the generalized likelihood ratio (GLR) test follows as

$$
t_{\text {sens }}=\zeta^{T} \Sigma^{-1} \mathcal{J}_{t}^{T}\left(\mathcal{J}_{t}^{T} \Sigma^{-1} \mathcal{J}_{t}\right)^{-1} \mathcal{J}_{t}^{T} \Sigma^{-1} \zeta
$$

which is called sensitivity test. The test statistic $t_{\text {sens }}$ is $\chi^{2}$ distributed with non-centrality parameter $\delta_{t}^{T} F_{t, t} \delta_{t}$. For making decision about the damage location, the test variable is compared to a threshold.

Minmax tests: Instead of assuming the components of $\delta_{C}=0, \delta_{t}$ is tested using a residual blind to any change in $\delta_{C}$. This is achieved by a projection orthogonal to the space defined by $\delta_{C}$, also known as minmax test, as follows. Define the partial residuals $\zeta_{t}=\mathcal{J}_{t}^{T} \Sigma^{-1} \zeta, \zeta_{C}=\mathcal{J}_{C}^{T} \Sigma^{-1} \zeta$, and the robust residual $\zeta_{t}^{*}=\zeta_{t}-F_{t, t} F_{C, C}^{-1} \zeta$, whose mean is sensitive to changes $\delta_{t}$ but not to $\delta_{C}$. Testing $\delta_{t}=0$ against $\delta_{t} \neq 0$ with the GLR test yields

$$
t_{m m}=\zeta_{t}^{*} F_{t}^{*-1} \zeta_{t}^{*}
$$

where $F_{t}^{*}=F_{t, t}-F_{t, C} F_{C, C}^{-1} F_{C, t}$. The test statistic $t_{m m}$ is $\chi^{2}$ distributed with noncentrality parameter $\delta_{t}^{T} F_{t}^{*} \delta_{t}$.

\section{ESTIMATORS FOR DAMAGE QUANTIFICATION}

When damage is localized in the first step, the task for damage quantification is to estimate $\delta_{t}$ for the damaged components in the second step. Then, the parameter change follows as $\theta-\theta_{0}=\delta / \sqrt{N}$.

Sensitivity approach: An estimate of $\delta_{t}$ can be derived from the residual vector $\zeta$ as

$$
\hat{\delta}_{t}^{\text {sens }}=\left(\mathcal{J}_{t}^{T} \Sigma^{-1} \mathcal{J}_{t}\right)^{-1} \mathcal{J}_{t}^{T} \Sigma^{-1} \zeta
$$

where $\hat{\delta}_{t}^{\text {sens }} \sim \mathcal{N}\left(\delta_{t}, F_{t, t}^{-1}\right)$ for the assumption $\delta_{C}=0$.

Minmax approach: An estimate of $\delta_{t}$ based on minmax approach is

$$
\hat{\delta}_{t}^{m m}=F_{t}^{*-1} \zeta_{t}^{*} \sim \mathcal{N}\left(\delta_{t}, F_{t}^{*-1}\right) .
$$

\section{APPLICATION}

Both localization approaches and the quantification have been demonstrated on a four-story steel frame structure, the Yellow Frame (Figure 1), at the University of British Columbia. The structure is described in detail in $[8,9]$. It is supposed to show a linear vibration behavior. Twelve sensors are located on the structure, three at the north, south and west side in each floor (Figure 1(b)). For damaged and undamaged states, acceleration data containing 219,900 and 550,000 samples, respectively, at a sampling frequency of $1000 \mathrm{~Hz}$ were recorded. Damage is introduced by removing braces number 2 and 4 at the south side of the structure on the first floor.

After downsampling and decimation of the data by factor 5, seven well-estimated modes were obtained in the healthy and damaged states using subspace identification, together with their uncertainties. The identified frequencies are shown in Table I.

A finite element model of the structure is required to obtain $\mathcal{L}_{\text {model }}$ for the stress computation in the SDDLV approach, and to obtain the sensitivity of the modal parameters with respect to the structural parameters for the computation of the sensitivity $\mathcal{J}[8,9]$. 


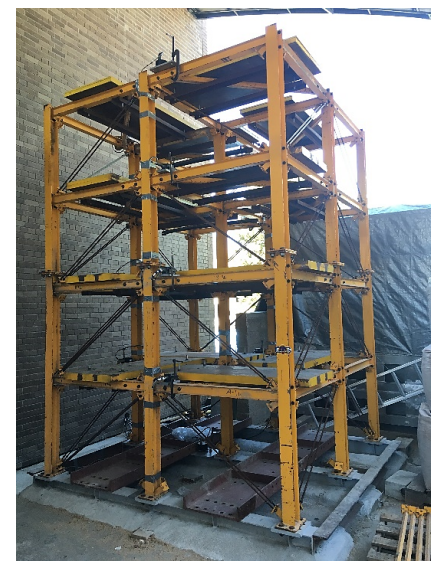

(a) Photo of the Yellow Frame structure (south-east corner)

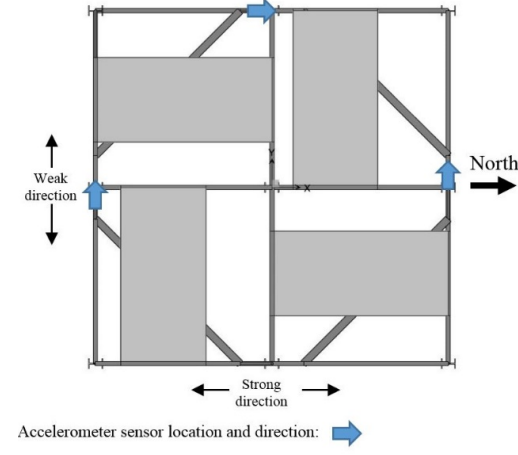

(b) Scaled plan of each level and location of sensors

TABLE I: Identified frequencies (in $\mathrm{Hz}$ ) of Yellow Frame.

\begin{tabular}{rrr}
\hline$\#$ & healthy & damaged \\
\hline 1 & 8.11 & 5.79 \\
2 & 8.71 & 7.97 \\
3 & 15.5 & 14.0 \\
4 & 21.4 & 19.9 \\
5 & 23.8 & 23.7 \\
6 & 31.2 & 30.6 \\
7 & 46.5 & 46.2 \\
\hline
\end{tabular}

Figure 1: Yellow Frame structure.

For simplicity, damage is located only in the braces. Hence, stress is only computed in the braces in the SDDLV approach, and parameter $\theta$ is defined only as Young's modulus of each brace in the sensitivity approach. Altogether, 32 brace elements are tested.

In both approaches, the transfer matrix difference and its covariance are computed based on the identified seven modes for two different choices of Laplace variables $s$, comprising one or several variables $s$ in the vicinity of the identified modes. Note that test values close to zero indicate potentially damaged elements in the SDDLV approach, and high values indicates the damaged elements in the sensitivity-based approaches.

\section{SDDLV approach: localization}

The computation of the stress and its covariance for the statistical evaluation in the $\chi_{t}^{2}$-tests is carried out for two different sets of $s$-values, each in the vicinity of the respective identified poles. First, one $s$-value is chosen with $s_{1}=-1+76 \mathbf{i}$, and second, two $s$-values are chosen as $s_{1}=-1+76 \mathrm{i}$ and $s_{2}=-1+30 \mathrm{i}$ for joint evaluation. To compare the ratios between the healthy and damaged elements, the computed values are normalized in the figures such that the smallest of the 32 values is 1 .

The estimated stress and its statistical evaluation is shown in Figures 2(a)-2(d). It can be seen that it is impossible to locate the damage at elements 2 and 4 with the estimated stress alone in (a) and (c), while its statistical evaluation in (b) and (d) correctly indicates the damage (smallest values). The additional $s$-value in (d) leads to a slightly better contrast to the damaged elements than in (b).

\section{Sensitivity-based approach: localization and quantification}

The damage indicators are shown in Figures 3 for each tested element using the transfer-matrix based residuals. In all cases, it is seen that the test statistic at the damaged elements shows the highest value, correctly localizing the damage at braces 2 and 4. However, in Figures 3(a) and (b) it can be seen that the tests also react strongly at undamaged elements using one $s$-value. By choosing three $s$-values in Figures 3(c) and 


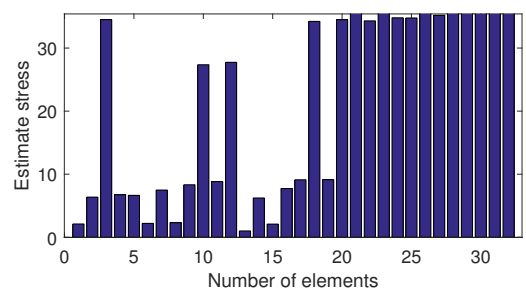

(a) Estimated stress, one $s$-value

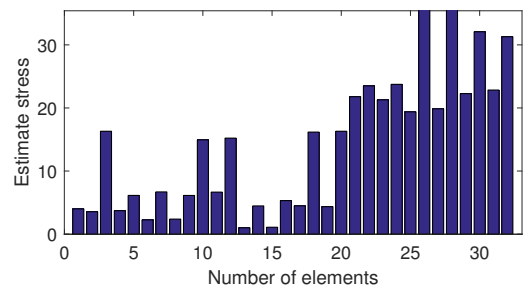

(c) Estimated stress, two $s$-values

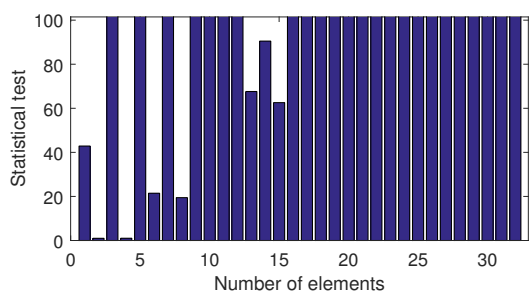

(b) Statistical test, one $s$-value

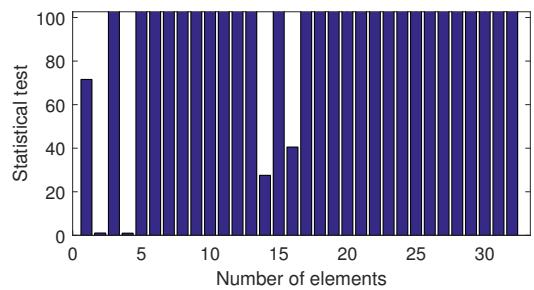

(d) Statistical test, two $s$-values

Figure 2: SDDLV-based damage localization: stress computation and statistical evaluation at one $s$-value $(-1+76 \mathbf{i})$ and two $s$-values $(-1+76 \mathbf{i},-1+30 \mathbf{i})$. Braces 2 and 4 are removed.

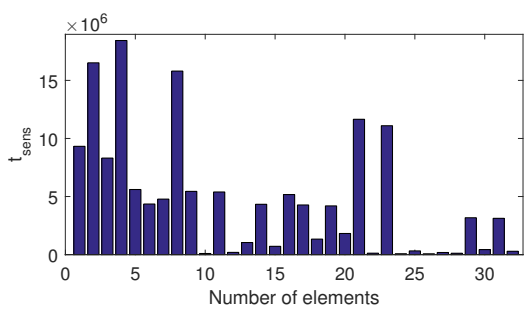

(a) Sensitivity tests, one $s$-value

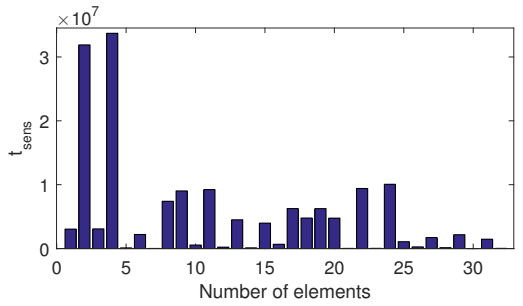

(c) Sensitivity tests, three $s$-values

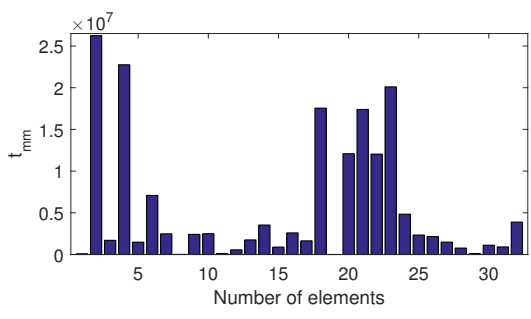

(b) Minmax tests, one $s$-value

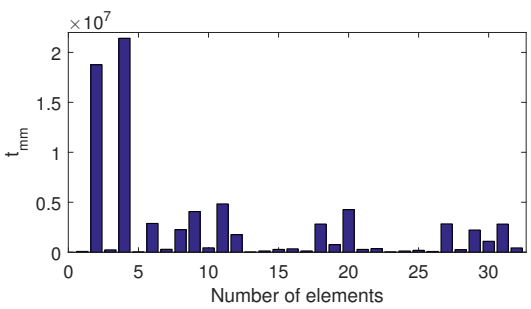

(d) Minmax tests, three $s$-values

Figure 3: Sensitivity-based damage localization: Sensitivity and minmax tests at one $s$ value $(-1+76 \mathbf{i})$ and three $s$-values $(-1+10 \mathbf{i},-1+250 \mathrm{i},-1+280 \mathbf{i})$. Braces 2 and 4 are removed.

(d) it can be seen that the performance is much better.

For the quantification of the damage extent, the values of $\hat{\delta}^{\text {sens }}$ and $\hat{\delta}^{m m}$ were estimated for the respective sensitivity and minmax approaches, and shown in Table II. Since the braces are removed, the stiffness loss should be $100 \%$. The minmax approach gets closer to this value than the sensitivity approach, however the overall quantification accuracy is not entirely satisfying. 
TABLE II: Quantification of damage extents (in \%)

\begin{tabular}{r|rr|rr}
\hline$\# s$-values & \multicolumn{2}{|c|}{1} & \multicolumn{2}{|c}{3} \\
\hline Damaged brace & 2 & 4 & 2 & 4 \\
\hline$\hat{\delta}^{\text {sens }}$ & 48.2 & 48.7 & 70.9 & 70.0 \\
$\hat{\delta}^{m m}$ & 86.0 & 94.2 & 77.1 & 102.2 \\
\hline
\end{tabular}

\section{CONCLUDING REMARKS}

In this paper, two transfer matrix based localization methods have been compared and achieved similar success on a real structure. The sensitivity based approach allows in addition the quantification of damage. The first quantification results are encouraging, however further understanding and work is needed to obtain more precise damage quantification estimates.

\section{ACKNOWLEDGMENTS}

Financial support from the Bretagne region is gratefully acknowledged.

\section{REFERENCES}

1. Farrar, C. and K. Worden. 2007. "An introduction to structural health monitoring," Philosophical Transactions of the Royal Society A: Mathematical, Physical and Engineering Sciences, 365(1851):303-315.

2. Bernal, D. 2010. "Load vectors for damage location in systems identified from operational loads," Journal of Engineering Mechanics, 136(1):31-39.

3. Döhler, M., L. Marin, D. Bernal, and L. Mevel. 2013. "Statistical decision making for damage localization with stochastic load vectors," Mechanical Systems and Signal Processing, 39(1-2):426-440.

4. Marin, L., M. Döhler, D. Bernal, and L. Mevel. 2015. "Robust statistical damage localization with stochastic load vectors," Structural Control and Health Monitoring, 22(3):557-573.

5. Bhuyan, M., M. Döhler, Y. Lecieux, L. Mevel, and F. Schoefs. 2017. "Statistical damage localization with stochastic load vectors using multiple mode sets," Structural Health Monitoring, in press.

6. Bernal, D. 2014. "Damage localization and quantification from the image of changes in flexibility," Journal of Engineering Mechanics, 140(2):279-286.

7. Döhler, M., L. Mevel, and Q. Zhang. 2016. "Fault detection, isolation and quantification from Gaussian residuals with application to structural damage diagnosis," Annual Reviews in Control, 42:244-256.

8. Allahdadian, S. 2017. Robust Statistical Subspace-Based Damage Assessment, Ph.D. thesis, University of British Columbia, Vancouver, Canada.

9. Allahdadian, S., M. Döhler, C. Ventura, and L. Mevel. 2017. "Damage localization of a real structure using the statistical subspace damage localization method," in Proc. 11th International Workshop on Structural Health Monitoring (IWSHM), Stanford, CA, USA.

10. Turek, M. and C. Ventura. 2007. "Development of a combined damage detection methodology," in Proc. 25th International Modal Analysis Conference (IMAC), Orlando, FL, USA.

11. Heylen, W., S. Lammens, and P. Sas. 1998. Modal Analysis Theory and Testing, Katholieke Universiteit Leuven, Belgium. 


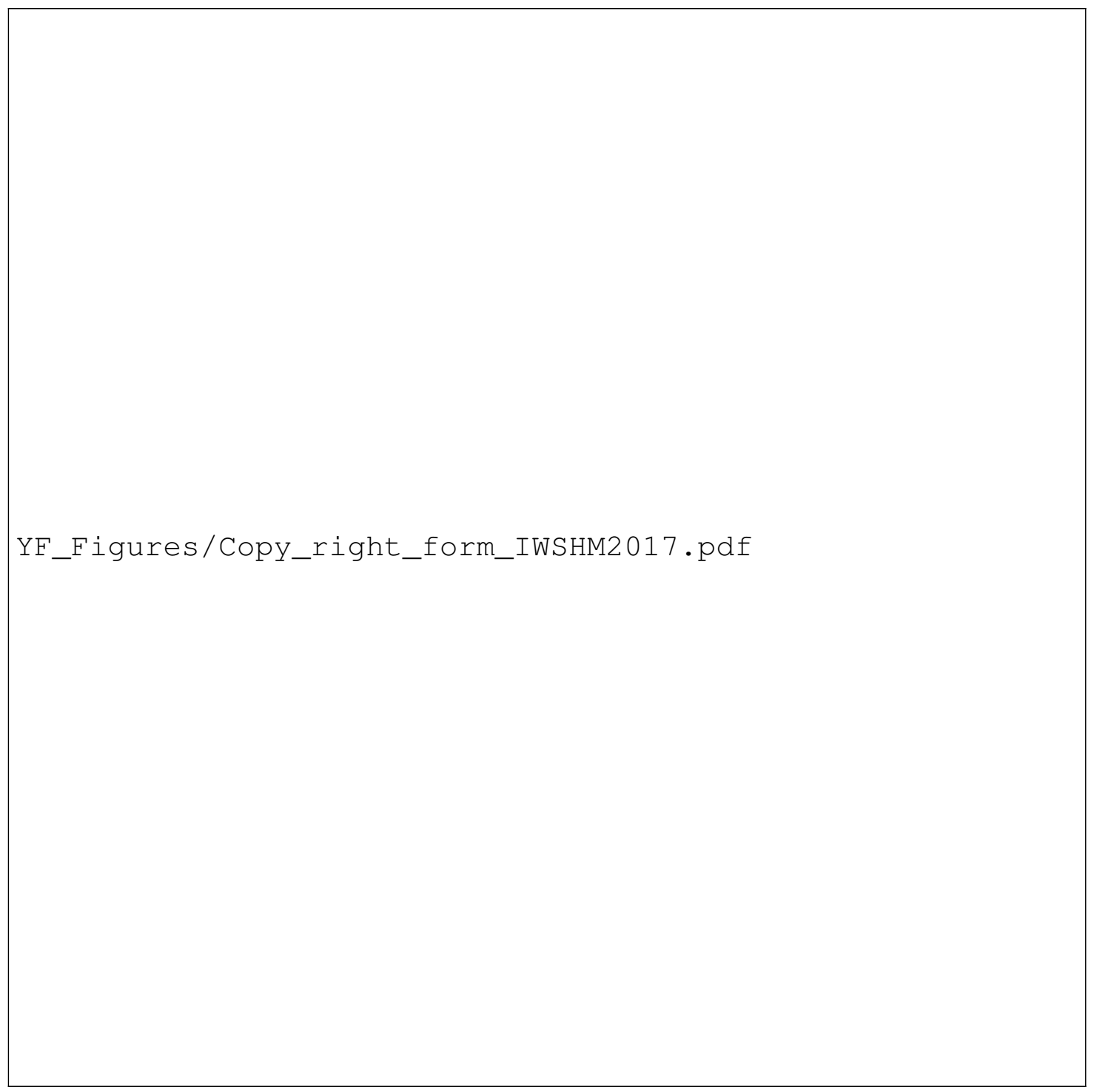

